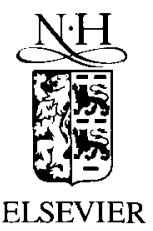

Invited Paper

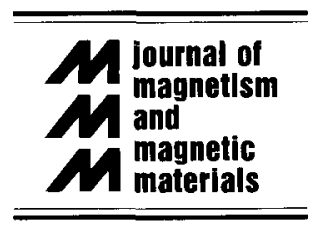

\title{
Effective $\mathrm{d}^{8}$ model: phase diagram
}

\author{
A.M. Oleś ${ }^{\mathrm{a}, *}$, L.F. Feiner ${ }^{\mathrm{b}}$, J. Zaanen ${ }^{\mathrm{c}}$ \\ ${ }^{a}$ Institute of Physics, Jagellonian University, Reymonta 4, PL-30059 Kraków, Poland \\ ${ }^{\mathrm{b}}$ Philips Research Labs, Prof. Holstlaan 4, NL-5656 AA Eindhoven, The Netherlands \\ ${ }^{c}$ Lorentz Institute for Theoretical Physics, Leiden University, P.O.B. 9506, NL-2300 RA Leiden, The Netherlands
}

\begin{abstract}
We present the phase diagram and excitations of an effective spin-orbital model derived for the $\mathrm{d}^{8}$ configuration of $\mathrm{Ni}$ ions in insulating $\mathrm{La}_{2} \mathrm{NiO}_{4}$. We identify a novel two-sublattice mixed-spin phase and argue that quantum fluctuations enhanced by the orbital degrees of freedom might stabilize a spin liquid in this regime.
\end{abstract}

Recently there is increasing experimental [1] and theoretical [2] evidence that orbital degrees of freedom play an important role in carrier propagation in doped transition metal oxides. Due to strong Coulomb interaction $U$ these materials are perhaps the best known examples of MottHubbard (charge-transfer) insulators in nature [3]. If the orbital degrees of freedom are indeed important, (i) one expects a more complex behaviour than that which follows from the $t-J$ model as the carriers are dressed by the excitonic excitations [4], and (ii) the present understanding of the undoped Mott-Hubbard insulators, based on the Heisenberg Hamiltonian, is quite unsatisfactory. Considering strongly correlated magnetic models one would like to understand: (i) whether the antiferromagnetic (AF) phases are significantly modified by the orbital degrees of freedom, and (ii) what other new magnetic phases could be realized in the presence of orbital excitations. In order to address the above questions in a concrete physical situation, we study in what follows the spin-orbital model derived for the $\mathrm{d}^{8}$ configuration of $\mathrm{Ni}$ ions in $\mathrm{La}_{2} \mathrm{NiO}_{4}$.

Starting from the multiband model for $\mathrm{NiO}_{2}$ planes of undoped $\mathrm{La}_{2} \mathrm{NiO}_{4}$ we derived an effective strong-coupling model. As in the case of $\mathrm{CuO}_{2}$ planes of $\mathrm{La}_{2} \mathrm{CuO}_{4}$ [5], the holes occupy the $\mathrm{e}_{\mathrm{g}}$ orbitals: $3 \mathrm{~d}_{x^{2}-y^{2}}(x)$ and $3 \mathrm{~d}_{3 z^{2}-r^{2}}(z)$. In the limit of large Coulomb interaction $U$ and large charge-transfer energy with respect to the $d-p$ hybridization, the relevant $\mathrm{d}^{8}$ configurations are the (high-spin) triplet $B_{i m}^{\dagger}$, and the (low-spin) singlet $A_{i}^{\dagger}$. Taking into account the virtual transitions $\mathrm{d}_{i}^{8} \mathrm{~d}_{j}^{8} \rightleftharpoons \mathrm{d}_{i}^{7} \mathrm{~d}_{j}^{9}$, where the ex-

\footnotetext{
* Corresponding author. Fax: +48-12-337086; email: amoles@jetta.if.uj.edu.pl.
}

cited $\mathrm{d}_{i}^{7}$ and $\mathrm{d}_{j}^{9}$ configurations are low-spin $(|x \sigma\rangle$ and $|x \uparrow x \downarrow z \sigma\rangle)$ doublets, we obtain the Hamiltonian, called $\mathrm{d}^{8}$ model,

$$
\begin{aligned}
H= & \sum_{\langle i j\rangle}\left[\left(S_{i}+(-1)^{\delta \cdot y} \sqrt{\alpha_{1}} T_{i}\right) \cdot\left(S_{j}+(-1)^{\delta \cdot y} \sqrt{\alpha_{1}} T_{j}\right)\right. \\
& \left.+\alpha_{2} n_{i A} n_{j A}\right]-E_{S} \sum_{i} n_{i A} .
\end{aligned}
$$

For convenience we are using dimensionless parameters normalized to the superexchange $J=16 t^{2} / \alpha_{0}^{2} U$, where $t$ is the (effective) hopping between $x$-orbitals, and $\alpha_{0} \simeq 3$ stands for the ratio of the $x-x$ and $x-z$ hopping elements. Further, $E_{S}=\left(E_{z}-3 J_{\mathrm{H}} / 2\right) J^{-1}$ is the singlet-triplet excitation energy, $\alpha_{1}=\alpha_{0} / 8, \alpha_{2}=1 / 4$, and $n_{i A}=A_{j}^{\dagger} A_{i}$ is the singlet number operator at site $i$. The spin operators $S_{i}$ are defined by the components of the triplet in the standard way, e.g. $S_{i}^{+}=\sqrt{2}\left(B_{i, 1}^{\dagger} B_{i, 0}+B_{i, 0}^{\dagger} B_{i,-1}\right)$. The pseudospin operators $\boldsymbol{T}_{i}$ are unusual and describe the transitions between the singlet and the components of the triplet which occur due to the mixing of the low- and high-spin sectors by the kinetic exchange, $T_{i}^{+}=\sqrt{2}\left(B_{i, 1}^{\dagger} A_{i}-A_{i}^{\dagger} B_{i,-1}\right), T_{i}^{z}$ $=-\left(B_{i, 0}^{\dagger} A_{i}+A_{i}^{\dagger} B_{i, 0}\right)$. The spin and pseudopin operators obey a dynamical so(4) algebra. If the factors $(-1)^{\delta \cdot y}$ were absent, $\alpha_{1}=1$, and $E_{S}=\alpha_{2}=0$, the model would have the full $\mathrm{SO}(4)$ symmetry. This symmetry is explicitly broken in a realistic system by the ligand fields, and (1) has only SU(2) spin rotational symmetry. Interestingly, the obtained formal structure is very similar (identical at $\alpha_{2}=$ 0 ) to the so-called singlet-triplet model for the rare-earth systems [6]. The physical origin of the latter model is quite different, however, as the dipolar ferromagnetic interaction between the angular momenta consists there of spin and pseudospin exchange, while the orbital momentum is quenched in our case and the states with different spins interact by the $\mathrm{AF}$ superexchange. 
We studied the phase diagram of the $d^{8}$ model (1) by solving the (classical) problem in mean-field theory (MFT). At lower values of $E_{S}$ the superexchange dominates, whereas the singlets are favoured by an increase of the singlet-triplet excitation energy $E_{S}$. Therefore, one finds the high-spin AF and [low-spin singlet (SS)] phase for small (large) $E_{S}$ (see Fig. 1). The AF high-spin phase is a two-sublattice Néel state, while in the SS phase all sites are occupied by the singlets. In between we identified a new phase, stabilized by the singlet-triplet transitions in (1), which could not be found using a classical spin parametrization [4], called mixed-spin (MS) phase. The energy is optimized by a quantum mixture of a singlet $\left(A_{i}\right.$ ) with the $S^{z}=0$ component ( $B_{i, 0}$ ) of the triplet,

$\Phi_{i}^{\dagger}=\cos \theta_{i} A_{i}^{\dagger}+\sin \theta_{i} B_{i, 0}^{\dagger}$,

with $\theta_{i}$ alternating between two sublattices $\left[\theta_{i}= \pm \theta\right.$ for $i \in A(i \in B)]$, and $\left\langle T_{i}^{z}\right\rangle \neq 0$ playing the role of the order parameter. The minimum energy of the MS phase is found at $\cos (2 \theta)=\left(E_{S}-2 \alpha_{2}\right) /\left(8 \alpha_{1}+2 \alpha_{2}\right)$, with the energy gain coming from the superexchange of pseudospins $\sim \alpha_{1}$. The phase transition between the AF and MS phase is first order, while the MS phase changes continuously into the SS phase.

We investigated the elementary excitations of the three above ground states in random phase approximation (RPA) by solving the equations of motion for the respective Green functions, such as $\left\langle\left\langle S_{i}^{+} \mid S_{j}^{-}\right\rangle\right\rangle$[7]. Using an extension of the RPA [8] we determined the renormalization of the order parameters and ground state energies in the considered phases. In the high-spin phase the renormalization of $\left\langle S_{i}^{z}\right\rangle$ is enhanced (compared to the Heisenberg model) by the orbital fluctuations (Fig. 2), in particular close to the transition to the MS phase. This reduction of $\left\langle S_{i}^{z}\right\rangle$ is similar, but not quite as strong, as in the similar spin-orbital $\mathrm{d}^{9}$ model [8]. The renormalization of $\left\langle n_{i A}\right\rangle$ in the MS and SS phase is much weaker. With increasing $E_{S}$ the triplet-singlet fluctuations increase, and reduce the

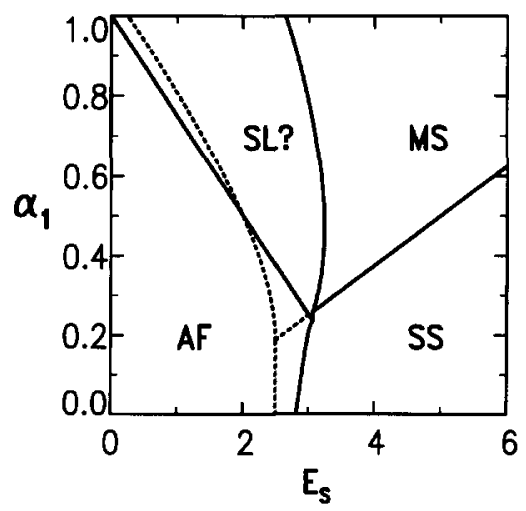

Fig. 1. Phase diagram of the $d^{8}$ model in MFT (dashed) and in RPA (full lines) as a function of $E_{S}$ and $\alpha_{1}$. The expected region of stability of the spin liquid (SL) phase is indicated.

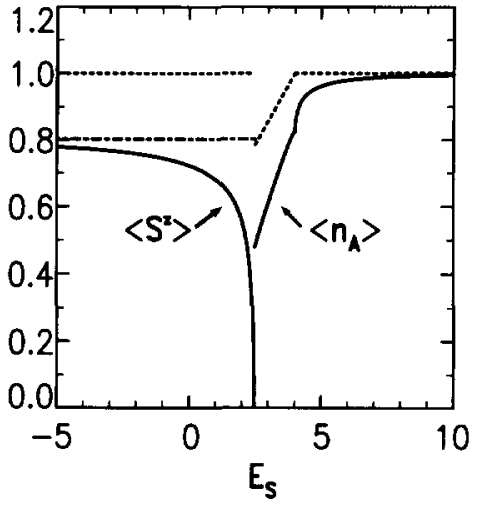

Fig. 2. $\left\langle S_{i}^{z}\right\rangle$ in the AF phase and $\left\langle n_{i A}\right\rangle$ in the MS and SS phases in MFT (dashed) and in RPA (full lines), as functions of $E_{S}$ for $\alpha_{0}=3$. In the Heisenberg model $\left\langle S_{i}^{z}\right\rangle=0.8$.

energy of the AF phase by an amount which is more than twice as large as the energy gain in the $S=1$ Heisenberg model. As the fluctuations (transverse and longitudinal) in the MS phase are much weaker, the AF phase wins and extends to lower energies. However, $\left\langle S^{z}\right\rangle \rightarrow 0$ and the AF phase collapses (at $E_{S} \simeq 2.5$ for $\alpha_{0}=3$ ), before the phase transition point to the MS phase is reached. Thus, we identify a region in the parameter space, where the fluctuations dominate the behaviour of the model and none of the simple phases considered in MFT is stable. This behaviour is unique for the AF superexchange and does not occur in the singlet-triplet model for the rare-earth materials [6].

In conclusion, we have found a new phase with mixed singlet-triplet order parameter. It signals the importance of singlet-triplet (orbital) fluctuations and is characterized by the increasing admixture of the triplet components when the AF phase is approached. It is expected that the orbital fluctuations which dominate the behaviour in the crossover regime might stabilize a novel and purely quantum spin liquid.

Acknowledgements: A.M.O. acknowledges the financial support by the European Community Contract ERBCIPACT920587 and the Committee of Scientific Research (KBN), Project No. 2038691 01, and J.Z. by the Royal Dutch Academy of Sciences (KNAW).

\section{References}

[1] J. van Elp et al., Phys. Rev. B 44 (1991) 6090 and 45 (1992) 1612; C.T. Chen et al., Phys. Rev. Lett. 68 (1992) 2543.

[2] J.H. Jefferson, H. Eskes and L.F. Feiner, Phys. Rev. B 45 (1992) 7959; J. Bała, A.M. Oleś, and J. Zaanen, Phys. Rev. Lett. 72 (1994) 2600.

[3] J. Zaanen, G.A. Sawatzky and J.W. Allen, Phys. Rev. Lett. 55 (1985) 418

[4] J. Zaanen and A.M. Oleś, Phys. Rev. B 48 (1993) 7197.

[5] J.B. Grant and A.K. McMahan, Phys. Rev. B 46 (1992) 8440.

[6] Y.Y. Hsieh and M. Blume, Phys. Rev. B 6 (1972) 2684.

[7] S.B. Haley and P. Erdös, Phys. Rev. B 5 (1972) 1106.

[8] L.F. Feiner, A.M. Oleś and J. Zaanen, J. Magn. Magn. Mater. 140-144 (1995) 1941 (these Proceedings). 